\title{
Multifocal histogenesis of a cystosarcoma phyllodes
}

\author{
R. SALM \\ From the Department of Histopathology, Royal Postgraduate Medical School, Hammersmith Hospital, \\ London W12 OHS, UK
}

SUMMARY An unusual multifocal cystosarcoma phyllodes is reported. It presented as a small, circumscribed tumour mass, which was associated with two diffuse neoplastic lesions which arose sequentially in two geographically separate parts of the mammary disc. Microscopically the tumour appeared to have been enlarging by the formation of isolated satellite tumour nodules within the adjacent normal breast tissue, which represents a third, though rarer way in which a cystosarcoma phyllodes may enlarge.

Cystosarcoma phyllodes (CSP) usually presents as a well-circumscribed mass, and some examples of this tumour are invested by a pseudocapsule due to compression of the adjacent breast tissues. Their microscopical appearances are similar to those of fibroadenomas, except for a greater cellularity of their fibrous component (Willis, 1967; McDivitt et al., 1968), and they are held to enlarge mainly by a process of expansion.

In contrast, the following case of CSP consisted only partly of a tumour with well-defined margins; microscopically it proved to extend much further. It was characterised by the presence of many scattered satellite foci, and by an unusual micronodular structure throughout, a tumour pattern which is virtually unknown.

\section{Case report}

A woman aged 50 years first presented with a small lump medial to the left nipple, and this was excised in April 1976. A few months later another lump had become palpable at some distance from the site of the previous excision, above and lateral to the nipple, and this was excised in November 1976. Because of tumour extension up to the excisional margin, this was followed a few days later by a simple mastectomy with removal of axillary lymph nodes. Because the opposite breast showed a general nodularity, and also in view of the multifocal nature of the tumour in the left breast, the surgeon considered that a simple mastectomy of the right breast was indicated, and this was carried out in January 1977.

Received for publication 8 February 1978

\section{MACROSCOPICAL FEATURES}

The two biopsy specimens of the left breast, and both mastectomy specimens, showed much nakedeye evidence of fibrocystic disease. The largest cyst of the first biopsy contained a polypoid ingrowth, about $1.4 \times 0.7 \mathrm{~cm}$, and the tissues adjacent to this cyst were of increased consistency. A similar firm, illdefined area was noted in the second biopsy specimen. In the area immediately adjoining the second biopsy site the left mastectomy specimen incorporated a fairly well-circumscribed, roughly spherical mass, $2.5 \mathrm{~cm}$ in diameter. The right breast contained many tense cysts, up to $2 \mathrm{~cm}$ across.

\section{MICROSCOPICAL EXAMINATION}

\section{Left breast, first biopsy}

The intracystic polypus consisted of a bulky fibrous tissue core, covered by intact and patchily hyperplastic epithelium. The subepithelial zones tended to be more cellular than the central parts, but mitotic activity was low.

The adjacent breast tissue showed the features of fibrocystic disease. In addition, there were a few scattered breast lobules with moderately dilated and elongated acini, separated from each other by proliferating fibroblasts with many mitotic figures (Fig. 1). A few of these acini, which were devoid of any surrounding elastic fibres, had become so elongated that they formed long tubular structures. Apart from these tubules, clearly arising from the breast lobules, the mammary tissues incorporated many isolated tubules of larger diameters which were not obviously related to any of the adjacent breast lobules. These also lacked elastic coats and were surrounded by thick collars of oedematous 


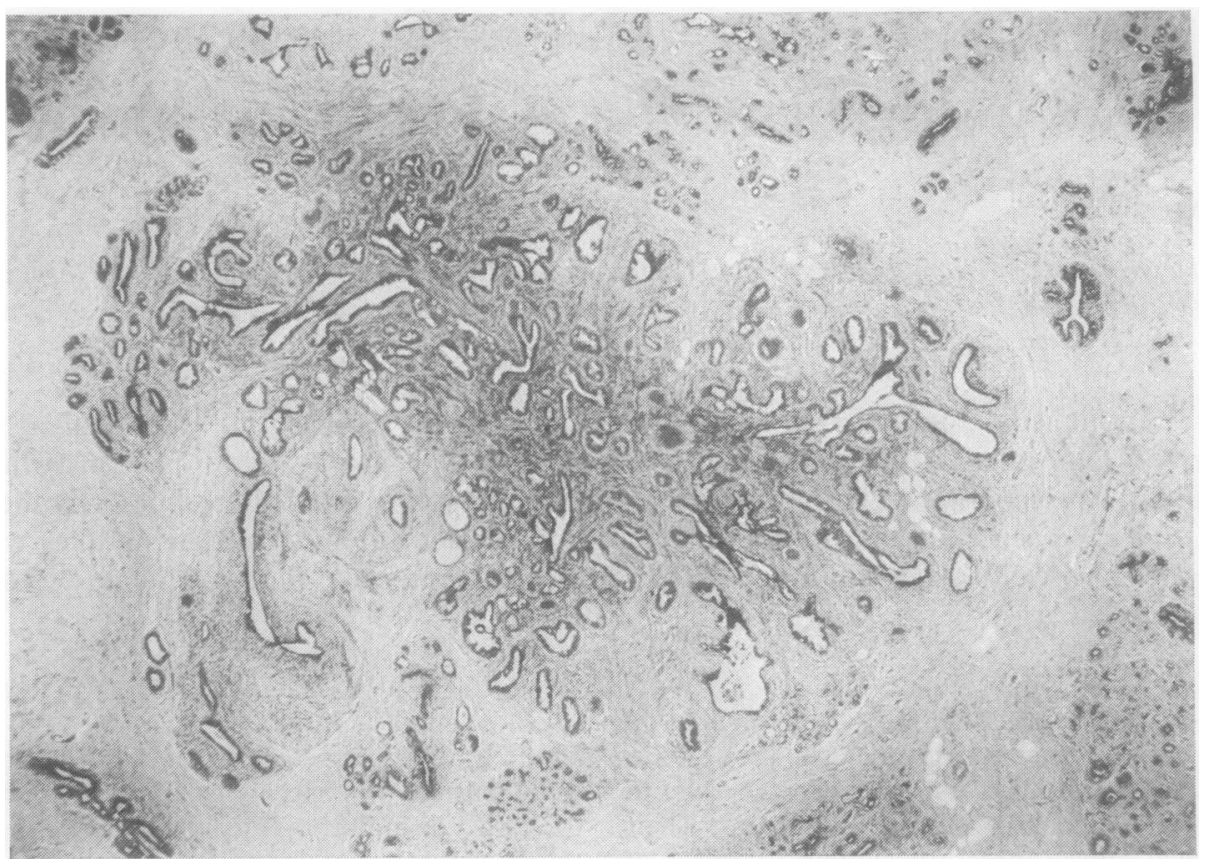

Fig. 1 Breast lobule with dilated, elongated, and tubular acini separated by proliferating fibroblasts. Haematoxylin and eosin $(H$ and $E) \times 30$.

fibrous tissue showing many mitotic figures; some of them were dilated, their lumina accommodating polypoidal protuberances.

\section{Left breast, second biopsy}

The features were essentially identical with those of the first biopsy. However, in an area about $2.5 \times 2$ $\mathrm{cm}$ the tumour nodules were more closely spaced, lying in a collagenous grid (Fig. 2), though with occasional interspersed groups of uninvolved breast lobules. In the more central parts these nodules were quite tightly packed without, however, having lost their individuality, silver impregnations showing each nodule to be clearly demarcated by a ring of reticulin fibres (Fig. 3).

Each single nodule consisted of a central duct-like structure, lacking any elastic investment, and these epithelial tubules were surrounded by a thick fibroblastic cuff with up to four mitoses per highpower field. The fibroblasts of many nodules were separated by much slightly alcianophilic oedema.

Another distinct feature, present throughout, was the occasionally very marked peritheliomatous arrangement of the immediately pericanalicular fibroblasts. These were placed parallel to each other and perpendicularly to the tubular epithelium, from which they were separated by a distinct, hyaline, eosinophilic zone (Fig. 4). Silver stains confirmed that this zone represented a thickened basement membrane to which the palisading fibroblasts appeared to be firmly attached. Thin reticulin fibres radiated outward from the thick basement membrane, between the fibroblasts, in parallel fashion (Fig. 5). In contrast, the more peripheral fibroblasts were lying haphazardly, or they were arranged in concentric fashion.

It was evident that extension of the neoplasm in the diffuse neoplastic areas had occurred in one of two ways. In the first place occasional breast ductules had become involved. Less often a larger breast duct had merged with a neoplastic nodule, resulting in the destruction of its wall and elastic coat; or an isolated duct had been affected by the neoplastic process, which was nevertheless identifiable as such, as its thick fibroblastic wall was still encased by an elastic coat.

However, more often newly formed, isolated neoplastic nodules were lying among somewhat atrophic but otherwise unremarkable breast tissue (Fig. 6), sometimes as far as $1 \mathrm{~cm}$ from the nearest, more compact tumour area (Fig. 7).

The tubular epithelium within the neoplastic nodules showed much patchy hyperplasia, especially when covering polypoidal ingrowths, but the 


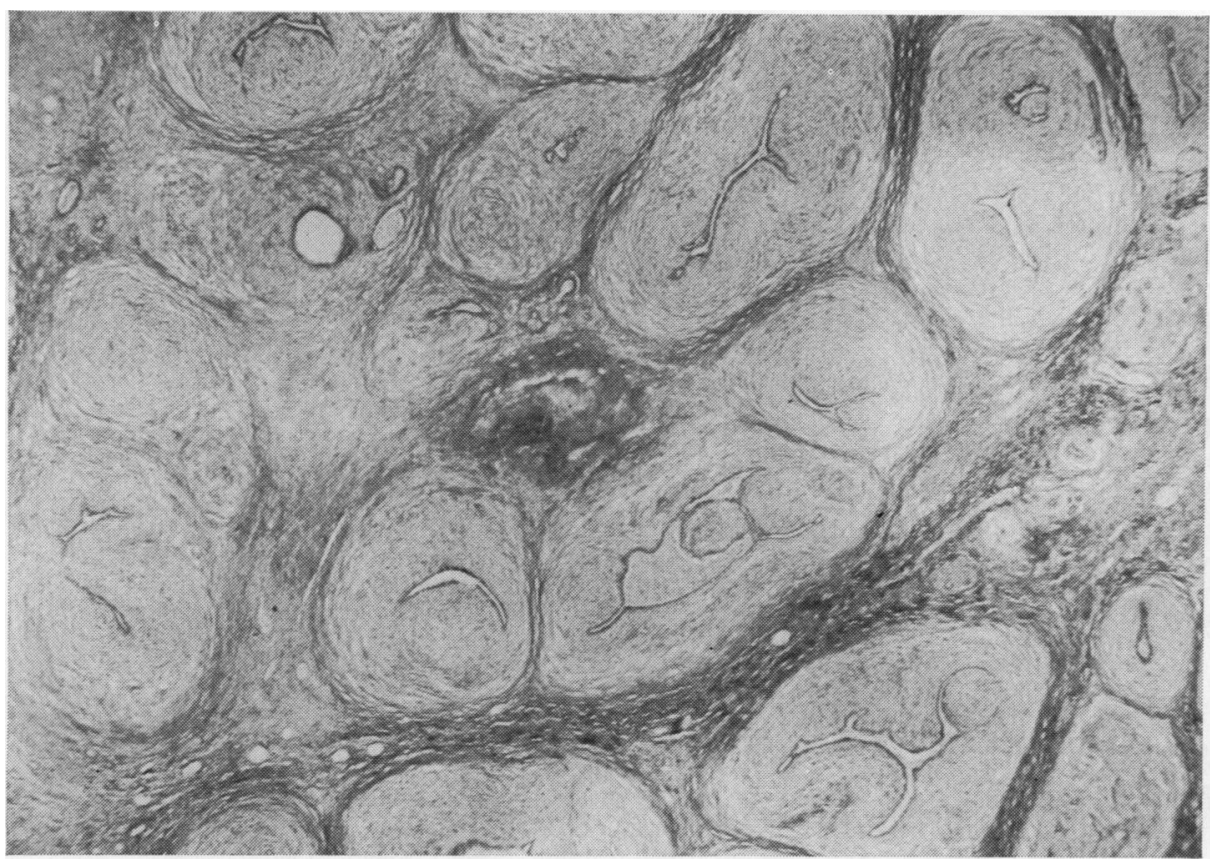

Fig. 2 Tumour nodules lying in a collagenous grid. van Gieson-elastic. $\times 30$.

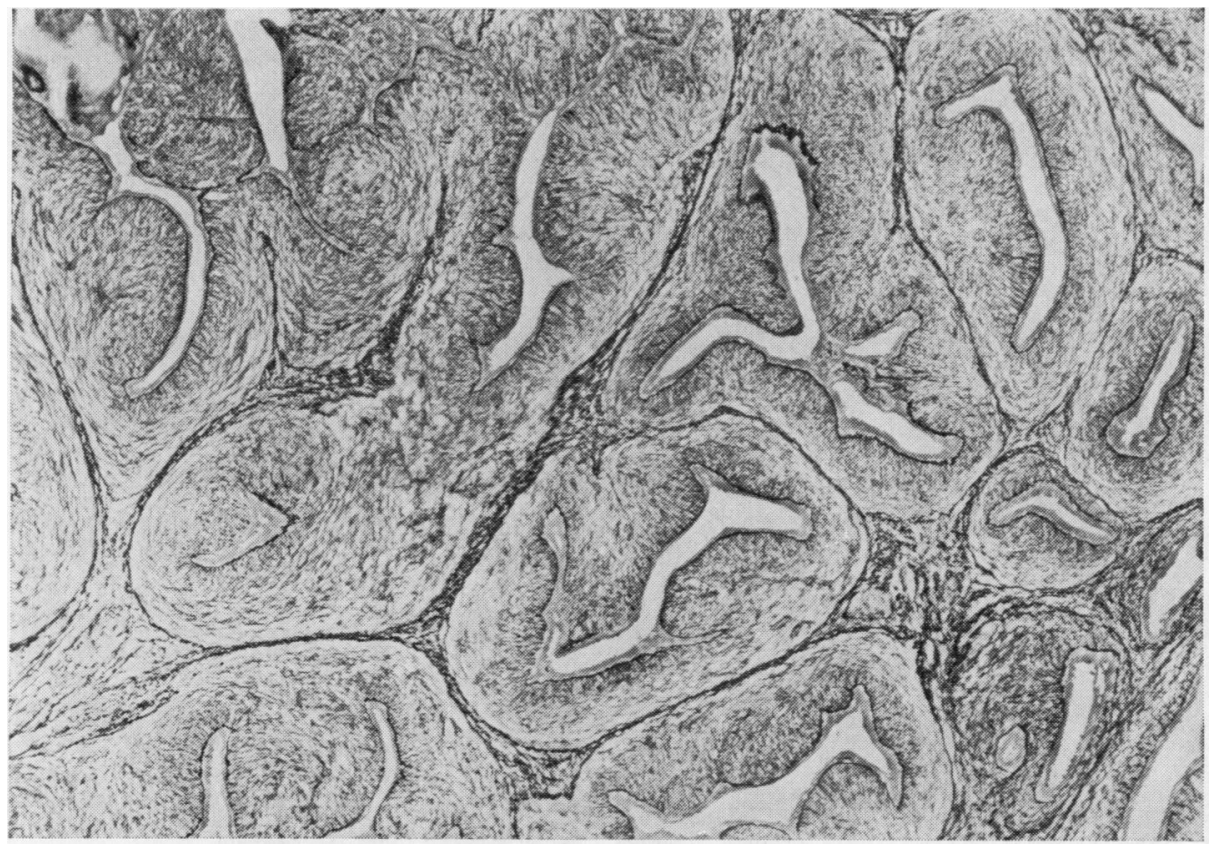

Fig. 3 Closely spaced tumour nodules sharply demarcated by surrounding reticulin fibres. Silver impregnation. $\times 30$. 


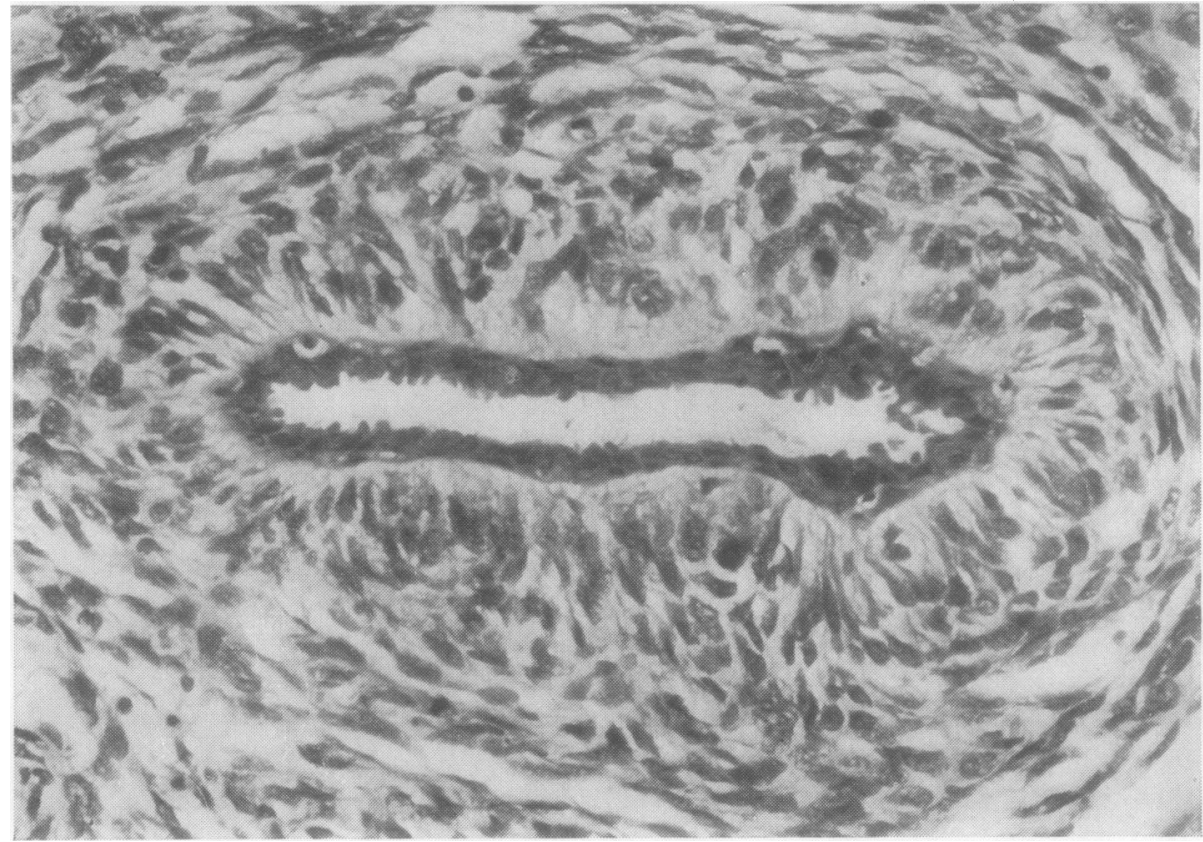

Fig. 4 Glandular tubule surrounded by rings of fibroblasts, three in mitosis. Perpendicularly arranged fibroblasts separated from epithelium by hyaline zone. $H$ and $E \times 30$.

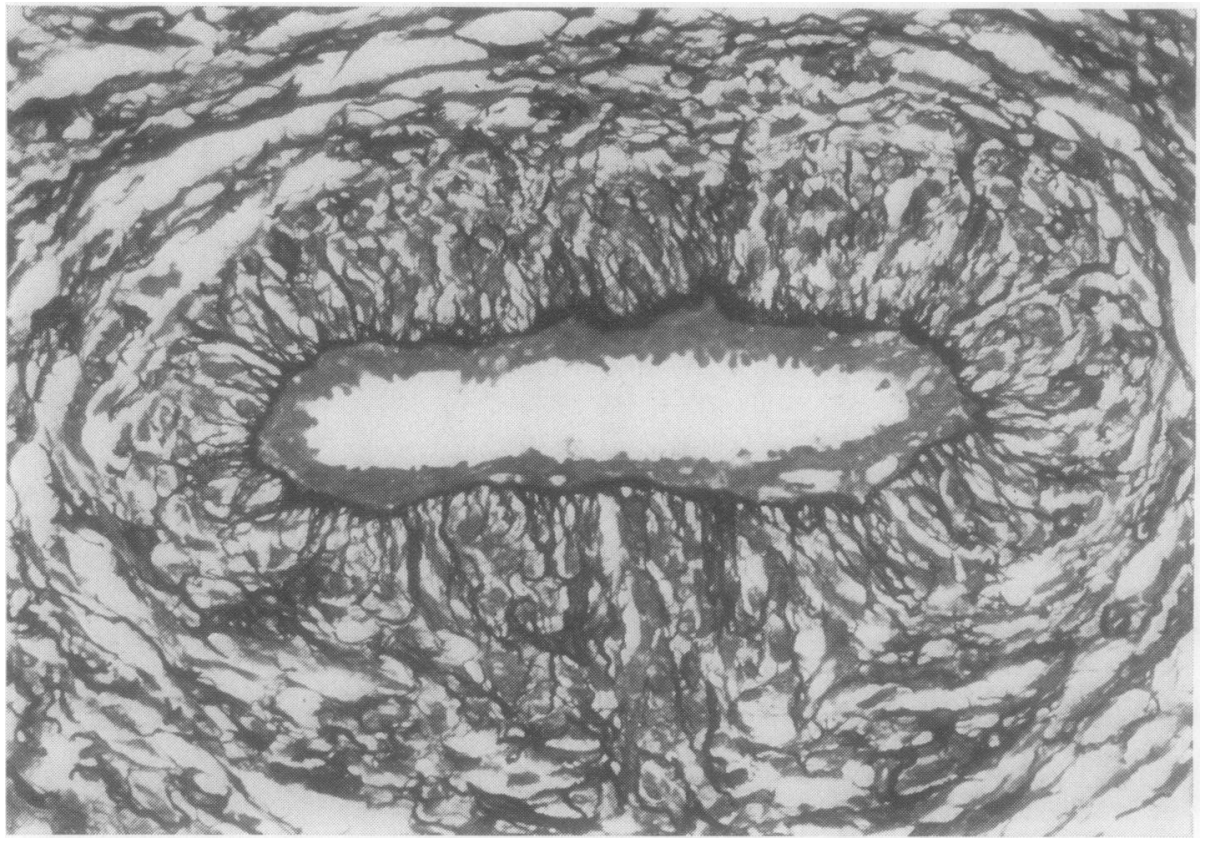

Fig. 5 Silver impregnation of adjacent section. Thickened basement membrane below epithelium from which parallel reticulin fibres irradiate outward between perpendicular fibroblasts. $\times 30$. 


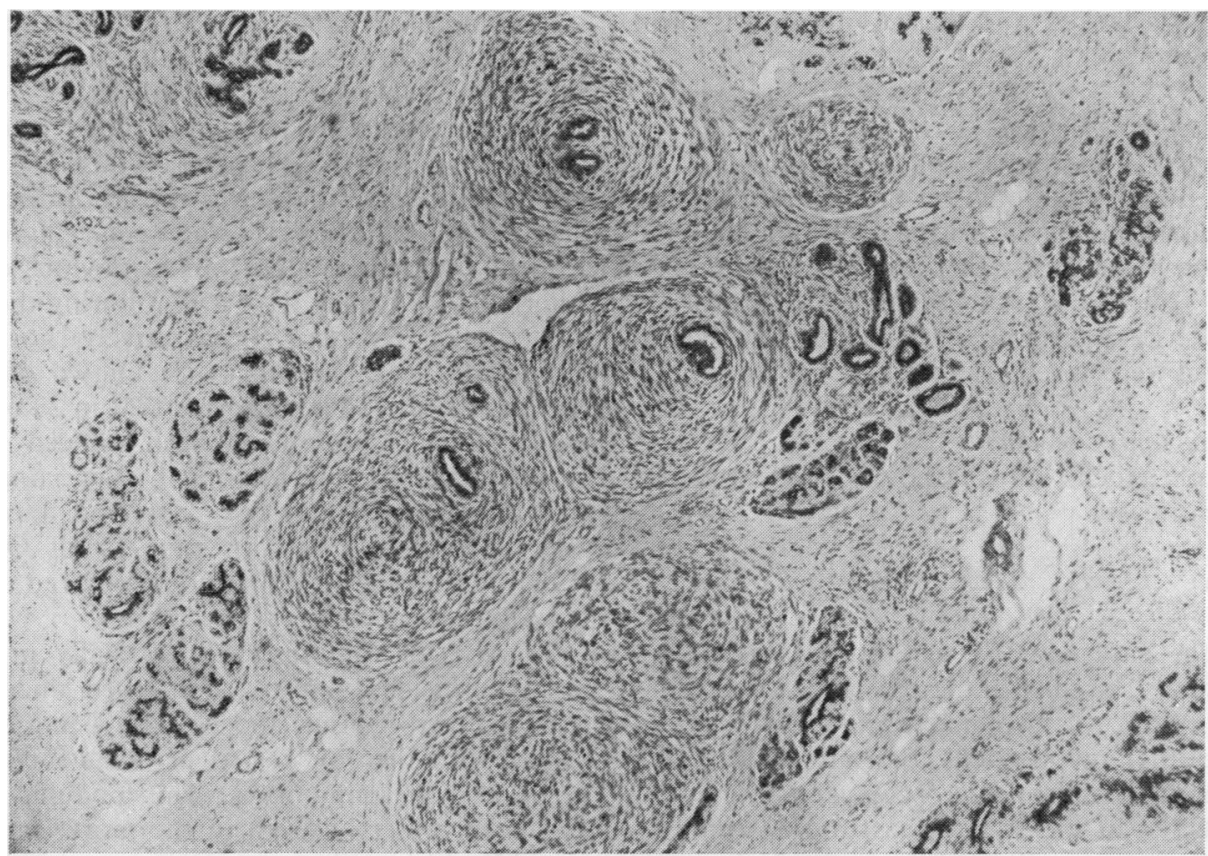

Fig. 6 Group of newly formed neoplastic nodules among atrophic but uninvolved breast lobules. $H$ and $E \times 30$.

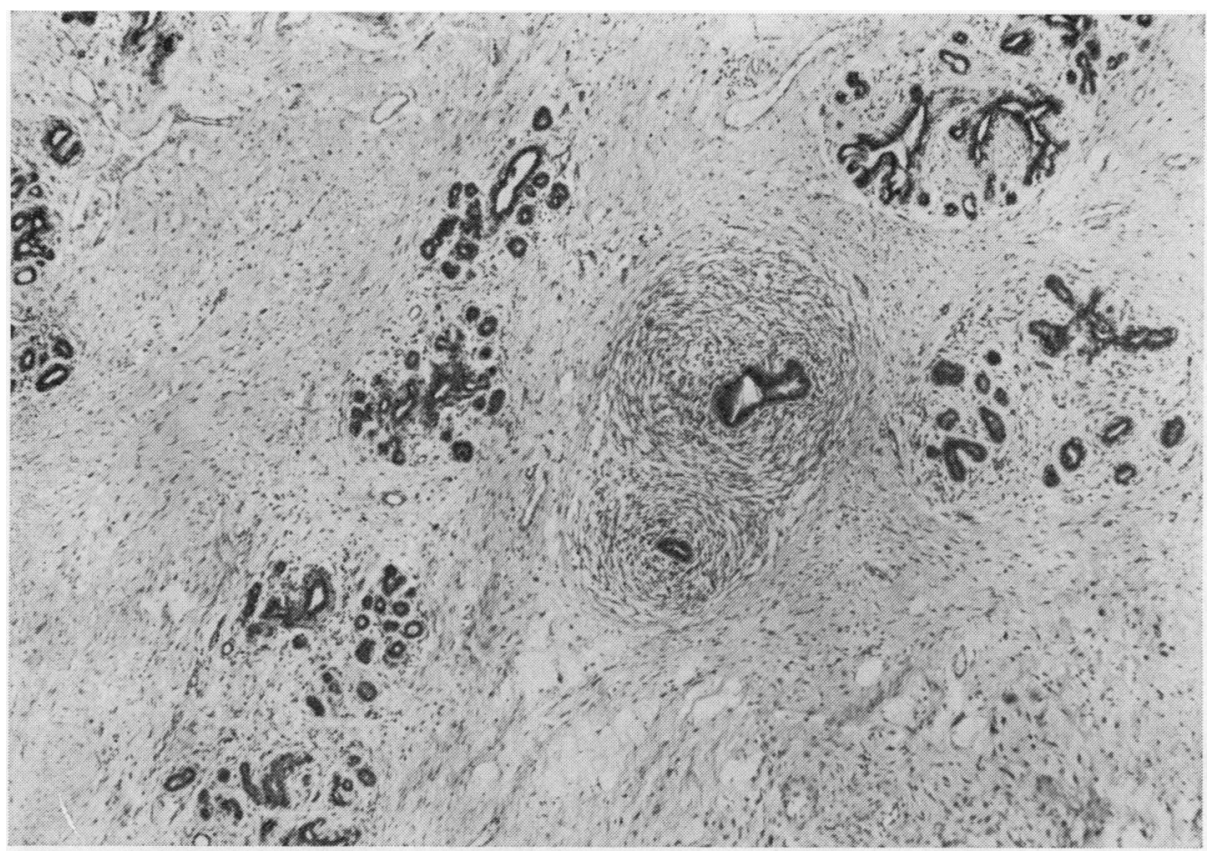

Fig. 7 Two neoplastic nodules present $1 \mathrm{~cm}$ from nearest more compact tumour area. $H$ and $E \times 30$. 
epithelial cells lacked mitotic activity and atypical features.

\section{Left mastectomy specimen}

The fibrocystic breast tissue contained a single small neoplastic nodule, as noted on naked-eye examination, with features similar to the central area of the second biopsy but with fairly well-defined margins (Fig. 8). Thus this mass met the conventional diagnostic criteria of a CSP. The six small lymph nodes found were unremarkable.

\section{Right mastectomy specimen}

The multiple blocks cut all showed the features of fibrocystic disease.

\section{Discussion}

The present case of CSP is unusual in several aspects-firstly, because of the presence, in addition to a small CSP, of two macroscopically separate areas of diffuse involvement, the nature of which became apparent only on microscopical examination. Multiple CSPs in the same breast are exceedingly rare. Minkowitz et al. (1968) reported the synchronous occurrence of one circumscribed malignant CSP, one circumscribed benign CSP, and two separate well-delimited fibroadenomas in a single breast of a woman aged 29 years.
Secondly, the tumours of the biopsy sites in the left breast were separated from each other in space and time. They were situated on either side of the nipple, separated by lactiferous ducts and normal breast tissue. It is, of course, possible that the second neoplastic focus was already present at the time of the first biopsy, though enlarging only subsequently and thereby becoming obvious clinically. Alternatively, it may have arisen during the ensuing five months. This is a comparatively short interval but would be in keeping with known data. Thus McDivitt et al. (1968) reported that in 28 of their 73 patients the tumours had been noticed only one month or less before treatment, suggesting a rapid growth potential, and they observed a recurrence after 11 months. And in case 15 of Oberman's (1965) series of CSP the tumour recurred after only three months.

Thirdly, all tumour foci, especially in the two biopsy excisions, showed a marked micronodular structure throughout, the nodules being separated from each other by a grid of collagenous fibrous tissue and reticulin fibres. This nodular structure had been preserved even in the more central, presumably older, and at any rate more compact areas. In the less crowded parts the tumour nodules were often separated by much uninvolved breast tissue.

Towards the periphery of the diffuse lesions there were breast lobules showing only a minor degree of

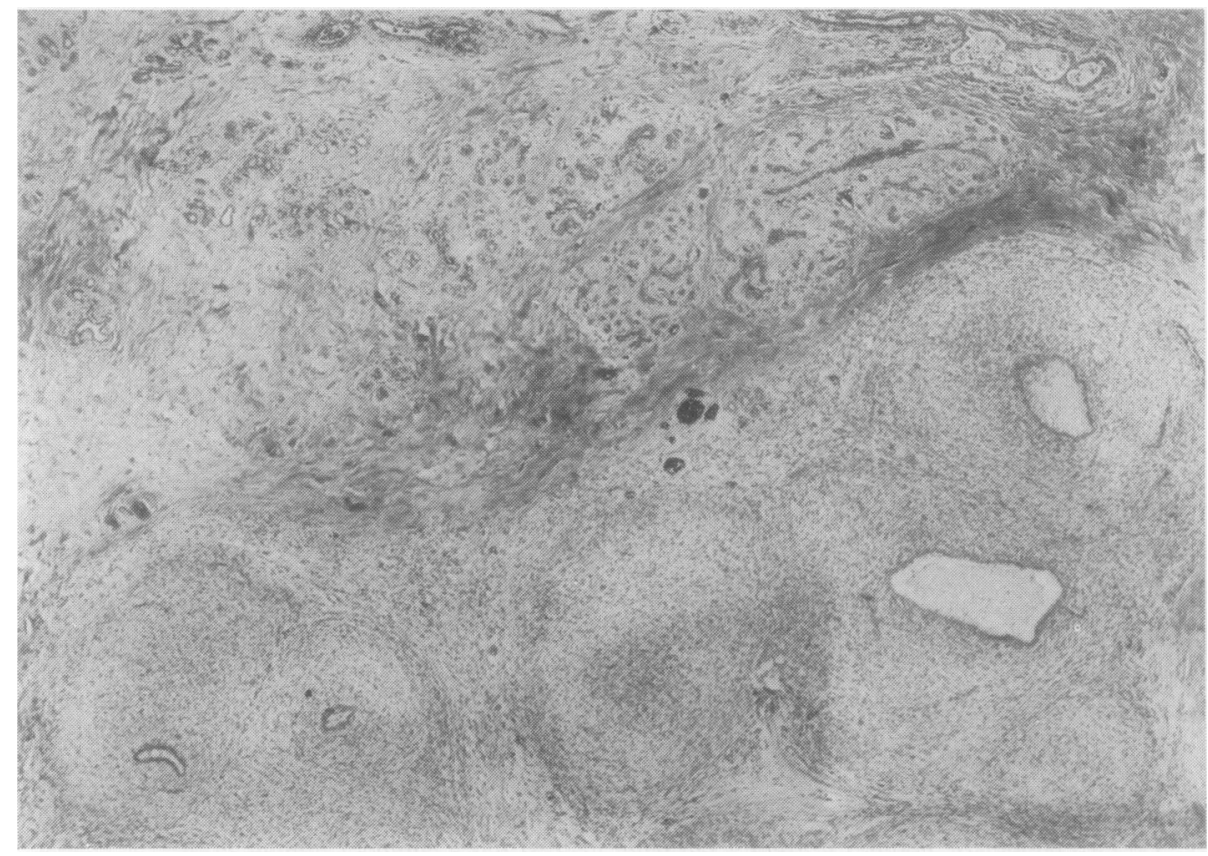

Fig. 8 Sharply defined periphery of small CSP in left mastectomy specimen. $H$ and $E \times 45$. 
neoplastic involvement. These were composed of enlarged and elongated acini separated by proliferating fibroblasts. Occasionally, however, extreme acinar elongation had produced tubular structures. Elsewhere the breast tissues incorporated many isolated duct-like tubules, sometimes lying in small groups, which were not obviously connected to any of the neighbouring breast lobules, and these were surrounded by thick fibroblastic cuffs. Both short and long tubules lacked elastic coats, a finding which suggests that most, if not all of them were of lobular origin. The pre-existing breast ducts had on the whole remained intact, except for an occasional duct which was merging with a tumour nodule, its wall and elastic coat being destroyed by proliferating fibroblasts; and there were a few isolated, thickened neoplastic ducts still contained within their elastic coats.

Many of the tumour nodules showed a peritheliomatous arrangement of the pericanalicular fibroblasts. These were separated from the epithelium by a distinct eosinophilic zone representing a thickened basement membrane, to which the palisading flbroblasts appeared to be firmly attached. These were supported by parallel reticulin fibres, which radiated outward from the basement membrane.

Finally, single, isolated tumour nodules were found up to $1 \mathrm{~cm}$ from the more compact tumour areas, indicating that tumour expansion had occurred by the induction of satellite tumours within the adjacent breast tissues. This must be regarded as a third mechanism by which CSP may enlarge, the other and more common modes being expansion from within, or by the extension of tumour projections from the periphery. It appears that McDonald and Harrington (1950) had previously observed this growth pattern, for they suggested, though they did not illustrate the process, that some of their cases of CSP had started from multiple foci throughout the breast, a mode of growth which, however, was subsequently denied by Treves and Sunderland (1951).

I acknowledge my gratitude to Professor H. K. Weinbren for affording me the hospitality of his department. Material and information were generously put at my disposal by Dr A. R. Kittermaster, Tunbridge Wells. I am indebted to Professor J. G. Azzopardi for much encouragement and advice. The photomicrography was the work of $\mathrm{Mr} \mathrm{W}$. Hinkes.

\section{References}

McDivitt, R. W., Stewart, F. W., and Berg, J. W. (1968). Tumors of the Breast (Atlas of Tumor Pathology, 2nd series, p. 119, Fascicle 2), Armed Forces Institute of Pathology, Washington, D.C.

McDonald, J. R., and Harrington, S. W. (1950). Giant fibro-adenoma of the breast-Cystosarcoma phyllodes. Annals of Surgery, 131, 243-251.

Minkowitz, S., Zeichner, M., DiMaio, V., and Nicastri, A. (1968). Cystosarcoma phyllodes: A unique case with multiple unilateral lesions and ipsilateral axillary metastasis. Journal of Pathology and Bacteriology, 96, 514-517.

Oberman, H. A. (1965). Cystosarcoma phyllodes. A clinicopathologic study of hypercellular periductal stromal neoplasms of the breast. Cancer, 18, 697-710.

Treves, N., and Sunderland, D. A. (1951). Cystosarcoma phyllodes of the breast: a malignant and a benign tumor. A clinicopathological study of seventy-seven cases. Cancer, 4, 1286-1332.

Willis, R. A. (1967). Pathology of Tumours, 4th edition, p. 217. Butterworths, London.

Requests for reprints to: Dr R. Salm, Histopathology Department, Royal Postgraduate Medical School, Hammersmith Hospital, Ducane Road, London W12 OHS. 